\title{
Experimental Contrast of the Effective Viscosity Determination Methods of Binary Flooding System
}

\author{
Li Liu \\ Key Laboratory of Enhanced Oil and Gas Recovery \\ of Ministry of Education of China \\ Northeast Petroleum University \\ Daqing, China \\ E-mail: liuliduoduo@163.com \\ Yanfu Pi \\ Key Laboratory of Enhanced Oil and Gas Recovery \\ of Ministry of Education of China \\ Northeast Petroleum University \\ Daqing, China \\ E-mail: piyanfu@163.com
}

\author{
Nan Chen \\ Petroleum Engineering \\ Northeast Petroleum University \\ Daqing, China \\ E-mail: 490984123@qq.com
}

\author{
Xue Wan \\ Petroleum Engineering \\ Northeast Petroleum University \\ Daqing, China \\ E-mail: 1585612604@qq.com
}

\begin{abstract}
For the development of oil and gas field, the effective viscosity determination of the viscoelastic fluid is always the problem of chemical flooding and there are technical problems to verify difficultly. The common methods include bulk phase viscosity method, the rheological curve method, and the drag coefficient method. This article tested and compared the methods respectively by adopting the binary system of fixed concentration. The results showed that using the method is more scientific that calculate the effective viscosity according to the curve transformation. The viscosity curve was obtained from the along sample tests in the long cores. For the experimental core with the length of $300 \mathrm{~mm}$ and permeability of $2500 \times 10-3 \mu \mathrm{m}$, the average of the injection viscosity and the viscosity of produced liquid is closer to the effective viscosity of binary system in the core.
\end{abstract}

Keywords-Offshore oil field; binary system; viscoelastic fluid; the effective viscosity; average viscosity method

\section{INTRODUCTION}

The sustained growth of production in offshore oil field has become an important part for the replacement of the oil production. With the increase of difficulty of exploration and the efficient development of offshore oil and gas resources, it is imperative to develop techniques to greatly improve the oil recovery in offshore oil field [12]. With the successful implementation of chemical flooding of onshore oilfield, all kinds of chemical flooding technology have been mature, especially the large-scale industrial application of polymer flooding. Chemical flooding technology has been developed gradually to the offshore oilfield with complex reservoirs, tough conditions of injection agents, high risk [3-5]. Polymer flooding and binary flooding as the most potential chemical flooding technology is the first choice to the offshore oil field after the water flooding.

This article chooses the binary system to study which contains the AP-P5 polymer and the BH-M2 surface active agent, which is based on the indoor experiment of the chemical flooding after polymer flooding of typical blocks in offshore oil field. The related production and research units had researched the related parameters of the binary system [6], which had been applied in other blocks of offshore oil field. The performance of the reagent is relatively good. In this article, polymer solution concentration in the binary system is $1750 \mathrm{mg} / 1$, and selected surface active agent concentration is $0.2 \%$. All experimental binary system formula adopts the component concentration in this article.

\section{THE EXPERIMENT PART}

\section{A. Experimental Apparatus and Experimental Conditions}

1. Incubator: accuracy within $1^{\circ} \mathrm{C}$;

2. Constant speed constant-flux pump: Xingda constantflux pump, constant speed displacement, can set different velocity;

3. Rotary vane vacuum pump: ZXZ-8 type, to swab air and filter;

4. Magnetic stirrer: used for dispensing solution;

5. Pressure sensor: with different range;

6. Brinell viscosity meter: used to determine the initial viscosity of polymer;

7. Electronic digital display scales: precision is $0.001 \mathrm{~g}$.

8. The experimental temperature: dispensing solutions is under the constant temperature of $45^{\circ} \mathrm{C}$, and the rest experiments are conducted under the condition of $65^{\circ} \mathrm{C}$.

9. Injection speed: at the seepage rate of $3 \mathrm{~m} / \mathrm{d}$ which reduced to the flow rate of the artificial homogeneous core with the width of $45 \mathrm{~mm}$ and the thickness of $45 \mathrm{~mm}$ is $1 \mathrm{ml} / \mathrm{min}$.

10. The experiment conducted with the method of constant speed. 


\section{B. The Preparation Methods of the Binary Composite System}

1. Mixture concentration of $5000 \mathrm{mg} / \mathrm{L}$ polymer mother liquid under the condition of $45^{\circ} \mathrm{C}$ firstly. The agitator rotational speed is set to $260 \mathrm{r} / \mathrm{min}$ when add the dry powder. As the samples get sticky, mix $4 \mathrm{~h}$ at the speed of $400 \mathrm{r} / \mathrm{min}$, seal the beaker with plastic film in the mixing process. Place the samples $6-8 \mathrm{~h}$ under the condition of the reservoir temperature $\left(65^{\circ} \mathrm{C}\right)$ after the mixing;

2. Dilute the surface active agent to $10 \%$ under the condition $45^{\circ} \mathrm{C}$;

3. Mix the polymer mother liquid, the surface active agent with the concentration of $10 \%$ and water of experimental simulation in proportion and stir the mixture $30 \mathrm{~min}$ under the condition of $45^{\circ} \mathrm{C}$ according to the concrete experimental plan.

4. Use warning blender to implement the pro-shear of purpose binary compound solution, the shear mode is level one (adjust the pointer to the line between 2 and 3), and the shear time is $20 \mathrm{~s}$.

\section{The Core for the Experiment}

The cores used in the experiments of the drag coefficient and the residual resistance coefficient are conventional standard cylindrical cores.

The cuboid artificial cores used in the verification tests, whose air permeability is $2500 \times 10^{-3} \mu^{2}$ and the size is $900 \times 45 \times 45 \mathrm{~mm}$. There lay pressure taps on the cores as shown in Fig. 1.

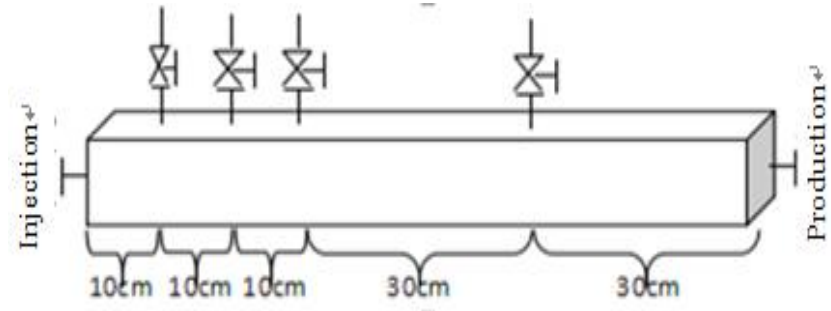

Figure 1. Effective viscosity test model for the core

\section{DIFFERENT METHODS OF VISCOSITY}

DETERMINATION [7-8] AND THE EXPERIMENTAL RESULTS AND COMPARISON

\section{A. The Rheological Curve Method}

In the process of polymer flooding, polymer solution viscosity and shear rate relate closely. What used directly in physical simulation experiment of polymer flooding is the seepage velocity, thus the conversion of the seepage velocity and the corresponding shear rate was needed. For a system, in order to determine the real velocity called $v$, firstly give an advance velocity called $v_{1}$. Put the values of the power-law index called $n$ of this system, the water permeability measurement called $\mathrm{K}_{\mathrm{w}}$ and the porosity called $\varphi$ of the experimental core into the formulas of displacement velocity and shear rate for the power law fluid which flows in the porous media. Shear rate can be obtained as follow:

$$
\gamma=\left[\frac{3 n+1}{4 n}\right]^{\frac{n}{n+1}} \times \frac{12 v}{\left(150 K_{\mathrm{w}} \varphi\right)^{\frac{1}{2}}}
$$

By the curvilinear regression of shear rate and viscosity of the solution, the formula of the change relation between viscosity and shear rate is the formula (2):

$$
\mu=K \cdot \gamma^{\alpha}
$$

The experimental measurement of oil in the offshore is shown in TABLE I :

TABLE I . THE MEASURED PARAMETERS OF POLYMER SOLUTION

\begin{tabular}{|c|c|c|}
\hline $\begin{array}{c}\text { Polymer solution } \\
\text { concentration (mg/l) }\end{array}$ & Index, $\boldsymbol{a}$ & Coefficient, \\
\hline 500 & -0.3426 & 14.304 \\
\hline 1000 & -0.371 & 32.663 \\
\hline 2000 & -0.503 & 155.39 \\
\hline
\end{tabular}

Fit the coefficient called $\mathrm{K}$ and the polymer solution concentration of the binary system (as shown in Fig. 2) by the formula (2), and obtain the formula as follow:

$$
y=6.558 \mathrm{e}^{0.0016 x}
$$

Type: $\mathrm{x}$ - polymer solution concentration.

Fit the index $(a)$ and the polymer solution concentration of the binary system (as shown in Fig. 3), the formula can be obtained:

$$
y=-5 E-08 x^{2}+2 E-05 x-0.3393
$$

So the formula of the change relation between viscosity and shear rate are:

$$
\mu=6.558 e^{0.0016 x} \cdot \gamma^{-5 E-08 x^{2}+2 E-05 x-0.3393}
$$

When the concentration of the binary system is that the polymer is $1750 \mathrm{mg} / \mathrm{l}$, the surface active agent is $2000 \mathrm{mg} / \mathrm{l}$, calculate the viscosity is $15.8 \mathrm{mPa} \cdot \mathrm{s}$.

Analysis of the Methods: the method can better reflect the shear effect of the core within porous medium to the displacing fluid, and it is the more commonly used viscosity processing method. 


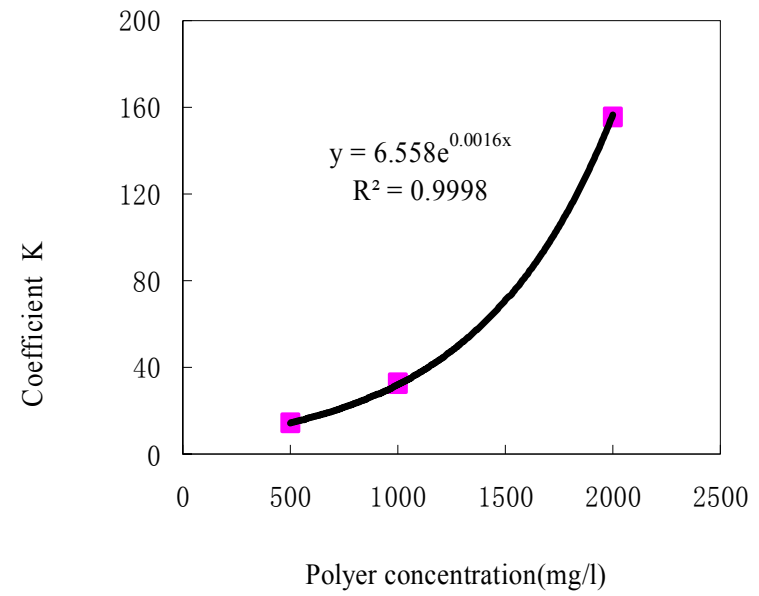

Figure 2. Relation of measured concentration and the coefficient of polymer solution

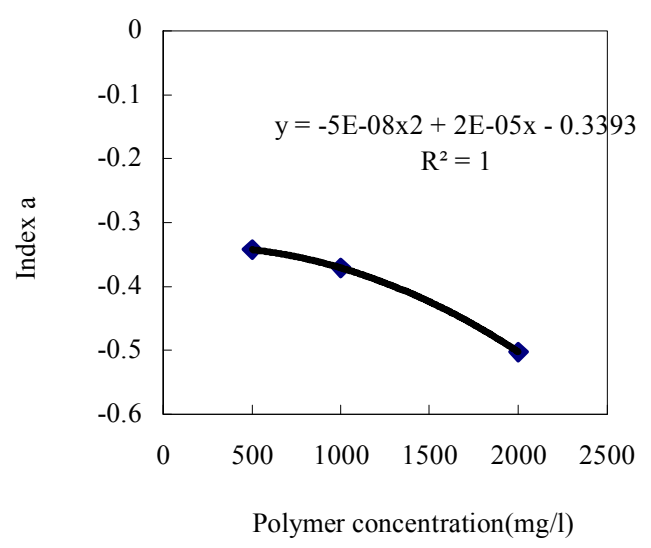

Figure 3. Relation of measured concentration and the index of polymer solution

However, this method only considered the shear effect on the viscosity, and ignored the adsorption of the displacement liquid in the core. The adsorption of the binary system must exist in the core, so this method is not applicable to process and analyze the data under the condition of oil in the offshore.

\section{B. Bulk Phase Viscosity Method}

The method is to calculate by putting the static viscosity value of the test before the injection of the binary composite system into the capillary number formula [9-10] directly. Analysis of the Method: the method is operated simply and calculated easily. There is no consideration of the viscosity loss in the core, so this method also is not applicable to process and analyze the data under the condition of oil in the offshore.

\section{Drag Coefficient Method}

The viscosity was tested by the conventional drag coefficient method which uses the following formula:

$$
\mu_{\mathrm{ef}}=\mu_{\mathrm{w}} R_{\mathrm{F}} / R_{\mathrm{RF}}
$$

In this paper, the selected binary system is used in the experiment of determination of the resistance coefficient and residual resistance coefficient, and the initial viscosity of the binary solution is $34.6 \mathrm{mPa} \cdot \mathrm{s}$. The permeability of the used core is the permeability of the reservoir of the typical offshore oil field. The permeability is $2500 \times 10^{-}$ ${ }^{3} \mu \mathrm{m}^{2}$. The drag coefficient of the binary solution is 40.45 . The residual resistance coefficient is 10.3. The calculated effective viscosity of the binary solution is 3.89 .

\section{Experiment of Core flow Test the Viscosity of the Binary System}

This article uses the method by laying the test point on the $90 \mathrm{~cm}$ long core to test the change of distance and the binary system viscosity (as shown in Fig. 1). The data graph is shown in Fig. 4.

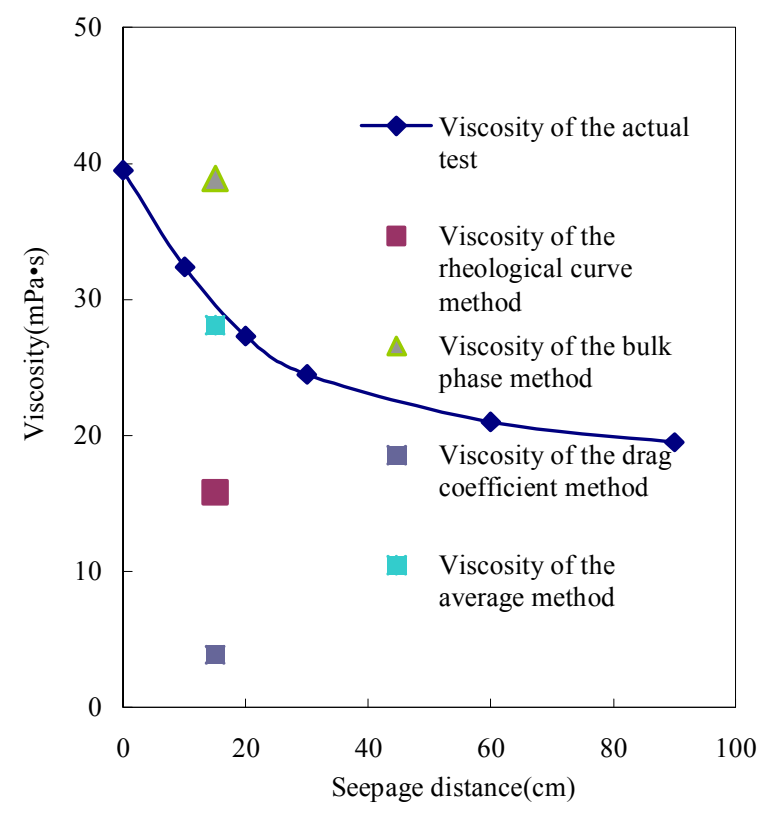

Figure 4 . The change of viscosity along the flow direction

The viscosity curve fitting formula is: $y=0.0039 X^{2}$ $0.554 \mathrm{X}+38.155$. Put $\mathrm{L}=15 \mathrm{~cm}$ in it, and get the viscosity of $30.72 \mathrm{mPa} \cdot \mathrm{s}$. At $30.72 \mathrm{mPa} \cdot \mathrm{s}$ as the effective viscosity in the process of core displacement, compare the value of viscosity calculated with the above methods. It is found that the calculation result with the average viscosity method is the most closed to the value.

The comprehensive analysis result of the above test methods is shown in Table II . 
TABLE II . COMPARISON OF THE ADVANTAGES AND DISADVANTAGES OF FITTING METHOD ON MEASURED DATA

\begin{tabular}{|c|c|c|c|}
\hline Methods & Advantages & Disadvantages & $\begin{array}{c}\text { Viscosity } \\
\text { value } \\
(\mathrm{mPa} \cdot \mathrm{s})\end{array}$ \\
\hline $\begin{array}{l}\text { The } \\
\text { rheological } \\
\text { curve } \\
\text { method }\end{array}$ & $\begin{array}{l}\text { The method can } \\
\text { better reflect the } \\
\text { shear effect of the } \\
\text { core within porous } \\
\text { medium to the } \\
\text { displacing fluid, } \\
\text { and it is the more } \\
\text { commonly used } \\
\text { viscosity } \\
\text { processing method }\end{array}$ & $\begin{array}{l}\text { It ignores the effects } \\
\text { of the adsorption of } \\
\text { the displacement } \\
\text { fluid in the core and } \\
\text { of Chromatographic } \\
\text { separation on } \\
\text { viscosity }\end{array}$ & 15.8 \\
\hline $\begin{array}{l}\text { Bulk phase } \\
\text { viscosity } \\
\text { method }\end{array}$ & $\begin{array}{l}\text { The method is } \\
\text { operated simply } \\
\text { and calculated } \\
\text { easily }\end{array}$ & $\begin{array}{l}\text { Without considering } \\
\text { the viscosity loss in } \\
\text { the core }\end{array}$ & 38.9 \\
\hline $\begin{array}{l}\text { Drag } \\
\text { coefficient } \\
\text { method }\end{array}$ & $\begin{array}{l}\text { Mature method, } \\
\text { considering the } \\
\text { shear and } \\
\text { adsorption of the } \\
\text { displacement } \\
\text { liquid in the core }\end{array}$ & $\begin{array}{c}\text { Pressure is not easy } \\
\text { to stabilize, and the } \\
\text { error is big }\end{array}$ & 3.89 \\
\hline $\begin{array}{l}\text { Average } \\
\text { viscosity } \\
\text { method }\end{array}$ & $\begin{array}{l}\text { The average } \\
\text { viscosity on behalf } \\
\text { of the effective } \\
\text { viscosity in the } \\
\text { process of } \\
\text { seepage, and it is } \\
\text { closed to the work } \\
\text { viscosity }\end{array}$ & $\begin{array}{l}\text { It is only used to one } \\
\text { by one measurement } \\
\text { for the experiment } \\
\text { and to } \\
\text { popularization and } \\
\text { can't be popularized } \\
\text { and applied }\end{array}$ & 28.1 \\
\hline
\end{tabular}

What can be seen from TABLE II is that the viscosity data calculated by the average method is more closed to the effective viscosity in the actual displacement process. While this approach is accurate relatively, the workload of the experimental method is huge. In the actual applications, each of the experimental binary systems needs to be tested. Because the permeability of the actual core is different, the core shear situation will be different.

\section{CONCLUSIONS}

1. The methods to determine the effective viscosity of the binary flooding system in the core contain the rheological curve method, phase viscosity method, drag coefficient method, etc. Through the comparison and verification, using the method is more scientific that calculate the effective viscosity according to the curve transformation, and the viscosity curve is obtained from the along sample tests of long cores.
2. The average of the injection viscosity and the viscosity of produced liquid is closer to the effective viscosity of binary system at the core for the experimental core with the length of $300 \mathrm{~mm}$ and permeability of $2500 \times 10^{-3} \mu \mathrm{m}$.

\section{ACKNOWLEDGEMENTS}

The research work was joint funding between the national major projects "Reservoir Research for Enhanced Oil Recovery Technology after Polymer Flooding in Offshore Oilfields" (2011ZX05024-004-14).

\section{REFERENCES}

[1] Zenglin Wang. Xinwang Song. Yangwen Zhu. Lixia Dou. Aizhong Xing and Wei Chen, "The Key Technology of Enhanced Oil Recovery for Binary Combination Flooding in Offshore Oilfield," Petroleum Geology and Oil Recovery, vol.21, Mar.2014, pp. 5-6.

[2] Chuanfei Wang, "The Evaluation Research of Reservoir Potential of Enhanced Oil Recovery for Chemical Flooding in Offshore Oilfield-Taking Suizhong 36-1 Reservoir as an Example," China University of Petroleum, 2010.

[3] Shanshan Jiang. Junru Yang. Fujie Sun. Xiansong Zhang. Haijiang Wang and Engao Tang, "The Injection Time and Field Application of Polymer for Offshore Oilfield," Offshore Oil, vol.29, Sep.2009, pp. 37-38. doi:10.3969/j.issn.1008-2336.2009.03.037.

[4] Xiansong Zhang. Engao Tang. Xiaoqing Xie and Wei Zheng, "The Research of Development Characteristics for Injection Polymer at Early and Injection Mode in Offshore," Journal of Oil and Gas, vol.25, Jul.2013, pp. 123-124. doi:10.3969/j.issn.10009752.2013.07.026.

[5] Kanyun Li, "The Formulating of Binary Combination Flooding in Offshore Oilfield," China University of Petroleum, 2011. doi:10.7666/d.y1876260.

[6] Xinmin Zhang. Yongjun Guo. Rusen Feng. Jianxin Liu. Jian Z hang. Xin Lv and Xujie Cao, "The Performance Study of the Binary Compound Drive System Suitable for the Suizhong 36-1 of Bohai Oilfields," Oil Field Chemical, vol.29, Sep.2012, pp. 322325.

[7] Yuanze Xu. Ping $\mathrm{Li}$ and Qingong He. "The Seepage Characteristics of PAM Aqueous Solution through the Micro Porous Medium," Oil Field Chemical, vol.1, Feb.1981, pp. 36-39.

[8] Ziheng Zhao translation, "The Rheological Problems in the Recovery of Oil and Gas," Beijing: Petroleum Industry Press, 1996.

[9] Shangqi Zhai, "The Effect of Capillary Number on Oil Displacement Efficiency of Polymer-surfactant System," Northeast Petroleum University, 2014

[10] Tao Dai. Kaihua Xi. Jialin Dai. Tongchao Lu. Jinbiao Yu. Yongqiang Ren. Yaozhong Yang and Aijie Cheng, "The Method of Binary Flooding Reservoir Simulation Based on the Capillary Number Interpolation," Journal of Shandong university (science edition), vol.47, Aug.2012, pp. 55-59. 